
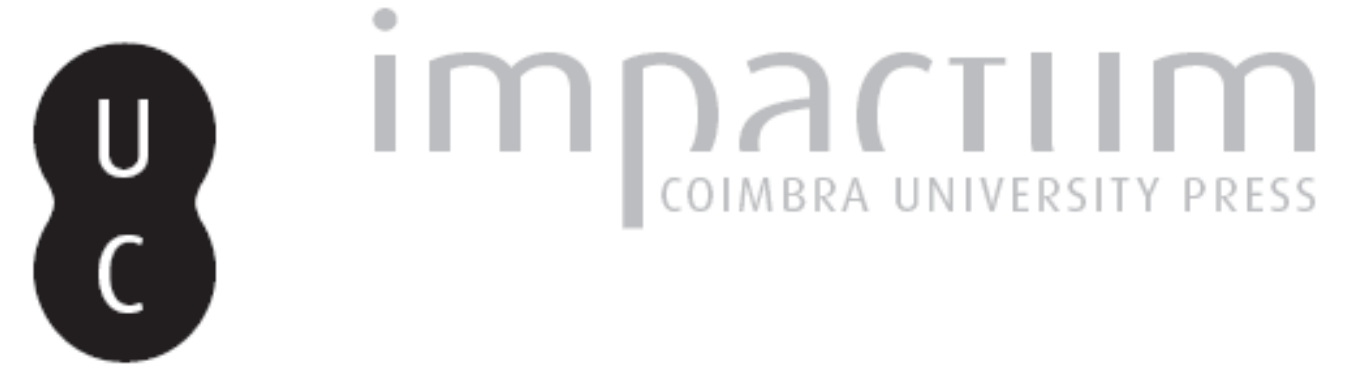

[Recensão a] Plato's Parmenides Reconsidered. By Mehmet Tabak

Autor(es): $\quad$ Ferrari, Franco

Publicado por: $\begin{aligned} & \text { Sociedade Internacional de Platonistas; Imprensa da Universidade de } \\ & \text { Coimbra }\end{aligned}$

URL

persistente:

URI:http://hdl.handle.net/10316.2/38751

DOI: $\quad$ DOI:http://dx.doi.org/10.14195/2183-4105_15_8

Accessed : $\quad$ 26-Apr-2023 06:35:15

A navegação consulta e descarregamento dos títulos inseridos nas Bibliotecas Digitais UC Digitalis, UC Pombalina e UC Impactum, pressupõem a aceitação plena e sem reservas dos Termos e Condições de Uso destas Bibliotecas Digitais, disponíveis em https://digitalis.uc.pt/pt-pt/termos.

Conforme exposto nos referidos Termos e Condições de Uso, o descarregamento de títulos de acesso restrito requer uma licença válida de autorização devendo o utilizador aceder ao(s) documento(s) a partir de um endereço de IP da instituição detentora da supramencionada licença.

Ao utilizador é apenas permitido o descarregamento para uso pessoal, pelo que o emprego do(s) título(s) descarregado(s) para outro fim, designadamente comercial, carece de autorização do respetivo autor ou editor da obra.

Na medida em que todas as obras da UC Digitalis se encontram protegidas pelo Código do Direito de Autor e Direitos Conexos e demais legislação aplicável, toda a cópia, parcial ou total, deste documento, nos casos em que é legalmente admitida, deverá conter ou fazer-se acompanhar por este aviso.

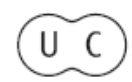


0

\section{Established 1989}

$\infty$ http://platosociety.org/

\section{Papers}

Thomas C. Brickhouse

Nicholas D. Smith

Socrates on the Emotions

Yosef Z. Liebersohn

Socrates, wake up! An analysis and exegesis of the "preface" in Plato's Crito (43a1-b9)

Nathalie Nercam

L'introduction problématique du Timée (17a-27a)

Christopher Moore

'Philosophy' in Plato's Phaedrus

Laura Candiotto

Plato's cosmological medicine in the discourse of Eryximachus in the Symposium. The responsibility of a harmonic technê

Anthony Hooper

Scaling the Ladder

Why the Final Step of the Lover's Ascent is a Generalizing Step

$\varangle$

Z
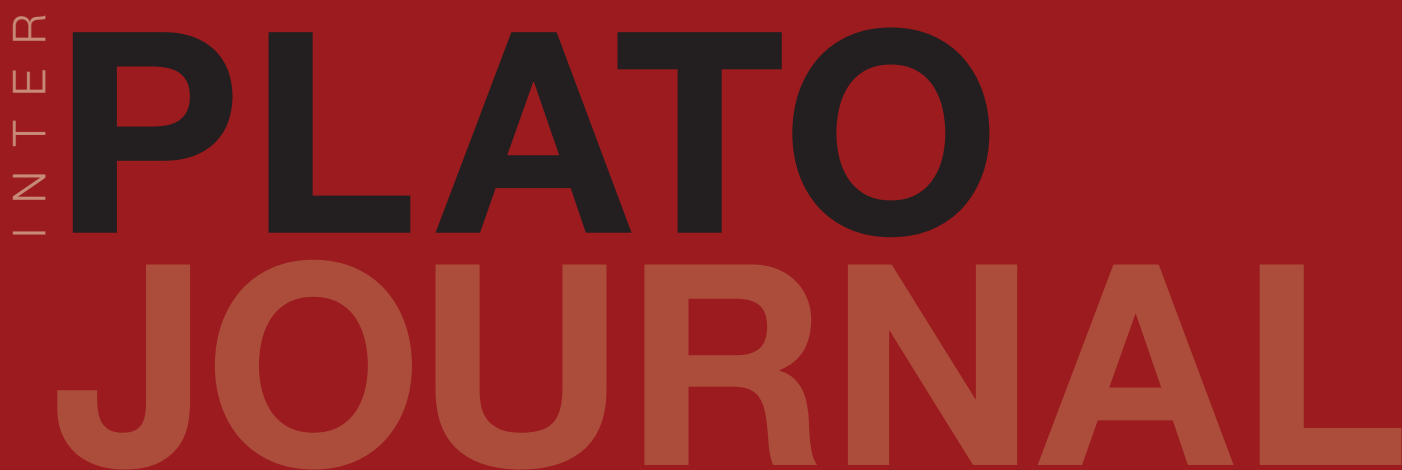

Société Platonicienne Internationale

Associazione Internazionale dei Platonisti

Sociedad Internacional de Platonistas

Internationale

Platon-Gesellschaft

Imprensa da

Universidade

de Coimbra

Coimbra

Universiy

Press 


\section{Plato's Parmenides Reconsidered. By Mehmet Tabak}

Palgrave Macmillan, New York 2015, pp. 229, £60.00.

\section{Franco Ferrari}

Università degli Studi di Salerno

fr.ferrari@unisa.it
L'intento perseguito dall'autore di questa coraggiosa monografia consiste nel dimostrare la sostanziale infondatezza della maniera più diffusa di interpretare il Parmenide platonico. In effetti, fatte salve le differenze, talora anche significative, che caratterizzano le indagini intorno a questo dialogo, sembra essersi imposta negli studi (soprattutto nell'ambito dell'area culturale anglofona) la convinzione che il Parmenide costituisca un punto di svolta fondamentale nel percorso filosofico di Platone. Una simile tendenza esegetica trova espressione sia nella diffusa convinzione che la prima parte del dialogo contenga una radicale auto-critica che Platone, per bocca di Parmenide, indirizza contro la versione della teoria delle idee contenuta nei dialoghi del periodo centrale, sia nel presupposto che l'esito teorico dell'esercizio dialettico che occupa la seconda parte del dialogo consista o nell'abbandono di questa teoria o in una sua sostanziale revisione. Gli studiosi che condividono un simile modo di interpretare il Parmenide sono poi propensi a considerare i dialoghi successivi, e in particolare il Teeteto e il Sofista, come lo sviluppo delle posizioni filosofiche formulate per la prima volta proprio nel Parmenide.

Il libro di Tabak si oppone fermamente a questo modo di vedere le cose. Lo studioso sostiene infatti che le obiezioni rivolte dal personaggio di Parmenide alla teoria delle idee esposta da Socrate sono del tutto inconsistenti dal punto di vista filosofico, che dunque esse non costituiscono affatto una sfida seria alla dottrina contenuta nei dialoghi del periodo centrale, e infine che la seconda parte del Parmenide non sviluppa una teoria positiva, alternativa alla concezione formulata da Socrate nei dialoghi precedenti e ribadita nella prima parte del Parmenide, ma contiene una sequenza di sofismi, fallacie e assurdità logiche, il cui solo scopo risiede nel dimostrare la contradditto- 
rietà e dunque l'assoluta insostenibilità delle posizioni eleatiche (e sofistiche).

E il caso di premettere che si tratta di un impianto esegetico che reputo nelle sue linee generali pienamente convincente, e la cui assunzione consentirebbe di affrontare con un atteggiamento ermeneutico corretto la lettura di questo misterioso dialogo.

Nel primo capitolo Tabak ricostruisce a partire dal Fedone, dal Cratilo e dalla Repubblica la concezione delle idee del periodo centrale. Gli assunti teorici fondamentali attengono alla natura assoluta e non qualificata di queste entità, che risultano auto-identiche, perfette, inalterabili, immobili e non mescolate alle cose sensibili, le cui caratteristiche dipendono dal fenomeno della "partecipazione" alle idee, le quali svolgono dunque una funzione di tipo causale; l'esistenza di una relazione tra le idee e le cose ordinarie non pregiudica in alcun modo la separazione delle idee, che va intesa nel senso dell'indipendenza e dell'autonomia ontologiche; le idee sono inoltre intelligibili, ossia conoscibili per mezzo della facoltà suprema dell'anima; infine, nei dialoghi centrali viene sviluppata un'epistemologia che riconduce i differenti gradi della conoscenza alla natura degli oggetti intorno ai quali la conoscenza stessa verte (pp. 5-28).

La concezione per mezzo della quale Socrate si propone di superare le aporie del molteplice avanzate da Zenone è, secondo Tabak, sostanzialmente identica alla dottrina dei dialoghi centrali (p. 29). Anche io sarei orientato ad avvicinare le due versioni, con una precisazione però, consistente nel richiamo alla circostanza che nella presentazione del Parmenide non compaiono accenni diretti all'anima e alla sua affinità (syngeneia) con il mondo delle idee, che costituisce uno degli elementi più significativi della theory of forms del Fedone, della Repubblica e del Fedro. Resta comunque in- discutibile che gli assunti fondamentali che definiscono la dottrina delle idee proposta da Socrate nella prima parte del Parmenide richiamino la concezione standard dei dialoghi sopra menzionati.

Prima di concentrarsi sulle obiezioni di Parmenide, Tabak fa una considerazione importante, che sembra in effetti supportare la sua interpretazione (pp. 29-35). Egli osserva, da un lato, che la figura di Zenone è agli occhi di Platone compromessa con la sofistica, dal momento che il suo allievo Pitodoro, il quale rappresenta l'anello di congiunzione tra gli eventi narrati e il racconto che Antifonte fa ai suoi ascoltatori, pagava per ascoltarne le lezioni, esattamente come facevano gli uditori dei sofisti (Alc. I 119 A). D’altro canto, neppure Parmenide, contrariamente a quanto solitamente si crede, risulta immune dalle riserve di Platone, il quale fa spesso seguire a dichiarazioni enfatiche (e non di rado ironiche) di ammirazione, smarcamenti netti e inequivoci dal pensiero del suo predecessore. Anche a proposito di questo punto ritengo che Tabak abbia assolutamente ragione; aggiungo che una più equilibrata valutazione dell'atteggiamento di Platone nei confronti di Parmenide non possa che giovare alla comprensione del pensiero di entrambi i filosofi.

A proposito delle due prime obiezioni che Parmenide rivolge a Platone, Tabak ha buon gioco nel dimostrare come esse presuppongano l'assunzione di un equiparazionismo ontologico del tutto estraneo alla concezione platonica delle idee. In effetti, tanto il celebre dilemma della partecipazione (che non può verificarsi né kata holon né kata mere), quanto l'ancora più noto argomento del 'terzo uomo' (che conduce all'ammissione di una molteplicità illimitata di forme), si fondano sull'illecito presupposto che le idee siano entità ontologicamente simili agli oggetti ordinari, ossia ai fenome- 
ni spazio-temporali (pp. 38-44). L'argomento con cui Parmenide si propone di confutare la partecipazione delle cose empiriche alle idee (131 A-E) funziona solo una volta che le idee siano concepite come realtà estese nello spazio, e perciò separate da se stesse (se i particolari partecipano all'idea nella sua interezza) e divisibili (se i particolari partecipano a singole parti dell'idea). Il suggerimento di Socrate, il quale propone di assimilare la presenza delle idee sui particolari partecipanti a quella del giorno, che è uno e identico ma contemporaneamente presente nella sua interezza in molti luoghi, consentirebbe di superare l'aporia, ma esso viene completamente frainteso da Parmenide. L'argomento che conduce alla proliferazione delle idee (132 A-B) si basa, invece, sull'errato (ossia non-platonico) presupposto che l'idea sia ciò che è a causa di qualcosa di separato da se stessa, vale a dire, per menzionare l'esempio del Parmenide, che l'idea della grandezza sia grande in virtù della partecipazione a un'altra idea del grande. Quanto simili presupposti siano estranei alla logica della concezione presentata da Platone nei dialoghi centrali è evidente a chiunque e non richiede ulteriori conferme.

Una certa attenzione merita invece una brillante osservazione di Tabak, il quale contrappone la maniera "platonica" di intendere l'espressione auto kath'hauto, che indica appunto l'indipendenza e l'autonomia ontologica delle idee, al fraintendimento nel quale incorre il personaggio di Parmenide, il quale concepisce l'ipseità delle idee nel senso della loro assoluta separazione, la quale finisce per comportarne la sostanziale inefficacia causale rispetto alle cose empiriche (p. 49). Se si volesse sviluppare e approfondire il ragionamento di Tabak, bisognerebbe constatare come Parmenide intenda in senso strettamente simmetrico la nozione di separazione, la quale invece com- porta, dal punto di vista di Platone, una relazione asimmetrica, in cui le idee sono, in quanto indipendenti, "separate", mentre i particolari fenomenici risultano, in quanto dipendenti causalmente dalle idee, non separati.

Nel complesso dunque il tentativo di Tabak di dimostrare la natura sofistica e inconsistente delle obiezioni di Parmenide risulta coronato da successo. È probabile che simili obiezioni rispecchino modi errati di concepire la dottrina di Platone, magari circolanti tra gli stessi allievi di quest'ultimo (e qui è quasi inevitabile menzionare Eudosso, il quale concepì la partecipazione in termini strettamente fisico-materiali, ossia come "mescolanza", mixis, adottando dunque un modello teorico molto simile a quello sulla base del quale il personaggio di Parmenide imposta il dilemma della partecipazione in $131 \mathrm{~A}-\mathrm{E})$.

Tutto ciò significa, evidentemente, che la concezione delle idee di Socrate, cioè quella contenuta nei dialoghi di mezzo, non viene scalfita da Parmenide e non ha nessun bisogno né di venire difesa, né tantomeno di essere rifondata. La seconda parte del dialogo non contiene dunque una "lezione" positiva, per la semplice ragione che il côté "positivo" del Parmenide è costituito dalla riproposizione della teoria delle idee dei dialoghi centrali.

Tabak porta poi un ulteriore elemento, questa volta drammatico, in favore della sua interpretazione. Si tratta della circostanza che nella seconda parte del dialogo Socrate viene rimpiazzato dal più giovane e inesperto dei presenti, quell'Aristotele destinato a diventare uno dei Trenta Tiranni. Un simile personaggio, un autentico yes-man, rappresenta, come Tabak osserva giustamente, "the perfect candidate to passively receive a barrage of sophisms from Parmenides" (p. 56). Il richiamo alla scarsa familiarità con la dialettica di questo personaggio non è nuovo, ma merita di venire valorizzato 
in considerazione della sua rilevanza nell'economia drammatica del dialogo.

Nel terzo capitolo Tabak affronta l'esame del misterioso esercizio contenuto nella seconda parte del dialogo (pp. 59-125). Come anticipato, si tratta per Tabak di una serie di sofismi tramite i quali Platone si propone di dimostrare l'inconsistenza e la contraddittorietà delle posizioni rivali, ossia di Parmenide e dei Sofisti, ai quali il filosofo eleate viene sostanzialmente accomunato. In particolare, secondo Tabak solo la terza serie di argomenti, ossia quella che esamina le conseguenze per gli altri nell'ipotesi che l'uno sia (157 B-159 B), può considerarsi compatibile con la teoria platonica delle idee, mentre la prima, la seconda, la quinta e la sesta non fanno che parodiare il punto di vista eleatico. La settima (e in maniera parziale la quinta) serie di deduzioni presuppongono la concezione protagorea, cioè la medesima dottrina che viene esposta e confutata nella prima parte del Teeteto, mentre l'ultima deduzione costituisce una critica a Protagora effettuata da un punto di vista parzialmente rispondente alla concezione di Platone (che sarebbe in qualche misura presente, sia pure in forma negativa, nella quarta ipotesi, quella relativa alle conseguenze per gli altri nel caso che l'uno risulti da essi completamente separato: $159 \mathrm{~B}-160 \mathrm{~B})$.

Si può naturalmente discutere sulla correttezza di una simile classificazione, che non sempre risulta del tutto convincente. Ma non c'è dubbio, almeno a mio avviso, che l'impianto generale dell'interpretazione della seconda parte del dialogo proposta da Tabak sia adeguato. In particolare mi sembra perfettamente rispondente all'andamento della gymnasia il richiamo al fatto che molti degli argomenti in essa contenuti presuppongono l'assunzione di una logica parmenidea, ossia di un'attitudine fisicista ed equiparazionista, sostanzialmente estranea alla prospettiva platonica. Per parte mia, mi sentirei di aggiungere che l'unico insegnamento che un simile esercizio è in grado di trasmettere a un ipotetico allievo risieda nell'impostazione fortemente deduttiva e consequenziale del ragionamento sviluppato da Parmenide, che rappresenta forse l'unica autentica eredità che Platone potrebbe avere ricavato dalla filosofia eleatica.

Il sincero apprezzamento che il recensore esprime per questo libro non lo esime dal lamentare l'eccesiva esiguità della bibliografia e il suo assoluto monolinguismo. Se Tabak avesse preso in considerazione con maggiore ampiezza la letteratura critica dedicata a questo dialogo, si sarebbe reso conto che molte delle tesi da lui sostenute non sono affatto nuove, e avrebbe potuto trovare ampia conferma alla sua interpretazione in alcuni studi relativamente recenti; per esempio a proposito dell'inconsistenza delle obiezioni di Parmenide e della natura non propositiva della seconda parte, avrebbe certamente potuto invocare a sostegno della sua lettura l'importante saggio di K. Dorter, The Theory of Forms and Parmenides I (pubblicato in J.P. Anton / A. Preus, Essays in ancient Philosophy, 3: Plato, New York 1989, pp. 183-202), o il prezioso volume di A. Graeser, Platons Parmenides (Stuttgart 2003), oppure ancora, si parva licet, l'edizione italiana del dialogo curata da chi scrive (Platone, Parmenide, Milano 2004). 